Natalie Jankowski*, Ekaterina Ivanova, Lea Wiehe and Michael Wahl

\title{
Long-term changes in technology acceptance of a robotic system in stroke treatment: a pilot study
}

https://doi.org/10.1515/cdbme-2020-2012

\begin{abstract}
Objectives: This pilot field study aimed to detect longterm changes in technology acceptance (TAM), user experience (UX) during use of an experimental demonstrator Bi-Manu-Interact (BMI) in stroke rehabilitation.

Methods: In 10 therapy sessions, patients performed a 20 min workout per session with the BMI. Patients with stroke were interviewed after the first (T0) and the 10th training session (T1) with a structured questionnaire about UX, TAM and potential to use the BMI at home. Nine patients ( $\mathrm{n}=4$ females) aged 33-78 years $(M=60.22 ; S D=13.17)$ participated in the study.

Results: After using the BMI no statistically significant differences have been found in UX and TAM (T0 vs. T1). But small to large effect sizes have been found which imply a practical relevance of the differences. The potential to use the BMI at home showed an increase in readiness for at $\mathrm{T} 1$ compared to T0.

Conclusion: Descriptive data suggest that familiarity gained through repetitive training sessions. The findings are coherent with previous studies.
\end{abstract}

Keywords: rehabilitation robotics; stroke rehabilitation; technology acceptance; user experience.

\section{Introduction}

Stroke is one of the major causes of acquired, permanent physical or mental impairments worldwide [1]. The World Health Organisation (WHO) estimates that this prevalence

*Corresponding author: Natalie Jankowski, Institut für Rehabilitationswissenschaften, Humboldt-Universität zu Berlin, Unter den Linden 6, 10099 Berlin, Germany,

E-mail: jankowna@hu-berlin.de

Ekaterina Ivanova, Department of Industrial Automation (FG IAT), Technical University Berlin, Berlin, Germany

Lea Wiehe and Michael Wahl, Institut für

Rehabilitationswissenschaften, Humboldt-Universität zu Berlin, Berlin, Germany rate will continue to increase in the future as a result of demographic change. Stroke patients usually suffer from severe hemiplegia, which leads to severe restrictions in everyday activities. A variety of therapeutic approaches exist to provide treatment for motor impairments after stroke $[2,3]$. Technical-assisted stroke rehabilitation is used in this context as a therapeutic procedure for the treatment of mobility [4]. The increasing use of mechanical devices and robotic systems is accompanied by a need for innovative system solutions to ensure a successful rehabilitation process.

\section{Robot-assisted arm therapy in stroke treatment}

Robot-assisted therapy has become a widely used therapeutic approach in stroke rehabilitation. A variety of clinical studies showed that patients benefit in recovery of upper limb from training with robotic systems [5-7]. The effectiveness can be explained by the degree of intensity and the number of repetitions per therapy session [7]. Most of the systems use an assist-as-needed strategy [6]. Patients are supported to such an extent that they can perform the desired exercise with maximum use of their remaining voluntary motor skills [6]. The main goal is the relearning of movements through exercise [6]. The use of multimodal feedback as well as training in virtual realities is attributed an increasingly central role [6, 7]. Studies showed that augmented feedback influence motor relearning [8] and mixed reality-based interventions have an effect on functions after stroke [9].

In the project "BeMobil - Regain Mobility and Motivity" the therapy robotic device Bi-Manu-Track (BMT) has been further developed for use in telerehabilitation environment. In order to prove a concept an experimental prototype Bi-Manu-Interact (BMI) by a Rehabilitation Robotics group, at the institute Industrial Automation Technology (IAT) of the TU Berlin. The BMI was tested in an experimental setup that is described below: Therapist and stroke patient sit opposite to each other and interact through the BMI. Each person receives visual feedback on a monitor about the quality of the movement [10]. 


\section{Technology acceptance}

In the mid-1990s, it was recognised that in order to improve user satisfaction and acceptance, other features of the technology had to be taken into account. These go beyond the classical functional application context and are related to the user's attitudes, needs, desires and abilities [11, 12]. In addition, Marcellini and colleagues (2000) refer to sociodemographic factors such as age, gender, level of education, health status and social conditions as predisposing variables in the context of technology acceptance, since they influence the way the technology is used [13].

One model that includes those factors is the Technology Acceptance Model (TAM), which was first developed by Davis and colleagues in 1989 [14]. The TAM is dedicated to the prediction of behavioural intention and the use of technology. A meta-analysis conducted by King and $\mathrm{He}$ (2006) with 88 studies on the application of the TAM in different areas (e.g. Internet, e-commerce) showed that it is a valid, robust and widely used model [15]. The authors of the TAM assume that the actual use of a technology is determined by the perceived benefit and ease of use [14]. Previous findings of technology acceptance confirm that with an increased experience in the use of a device, the influence on the perceived benefit and the intention to use decrease. This is due to the advantages and disadvantages of a technology are perceived in a more differentiated way [16].

\section{Research question and hypotheses}

Based on the presented research findings the present study focusses on a detailed analysis of TAM and UX of the BMI. Therefore, the central research question is "Are there differences in TAM, UX and the willingness to use the technology at home between the first (TO) and after the 10th (T1) training session with the BMI?"

The following hypotheses are derived: H1: T1-ratings in terms of UX show higher values than T0-ratings. H2: T1ratings in terms of TAM show higher values than T0ratings. H3: The willingness to use the BMI at home is higher at $\mathrm{T} 1$ than at $\mathrm{T} 0$.

\section{Methods}

The pilot field study was conducted from May to November 2017, with patients with stroke in a rehabilitation hospital near Berlin. The procedure was as follows: In 10 therapy sessions, patients performed a 20 min workout per session with the BMI. The training included five tasks in which the patient interacted with the system. The goal was to improve the movement guidance with the help of visualizations. Patients with stroke were interviewed with a structured questionnaire (self-developed and conducted in a standardized manner) on UX, TAM and potential home use after the first (T0) and 10th training session (T1). The interview was conducted after the training unit, in the examination room at the table in front of the system. The lateral side of the writing hand was often the half of the body with the motor function restrictions of the upper extremities, so that an independent completion of the questionnaire would not have been possible without support.

\section{Study design and sampling}

To answer the research question, a study design with a repeated measurement was applied, looking for differences for the variables UX, TAM and potential of home use within the same group of participants. Inclusion criteria for the study were adequate cognitive and language skills to participate in an interview. These skills were subjectively assessed by therapists on site. Exclusion criteria were the following: severe language and speech disorders, neglect, limitations in cognitive performance and decreased awareness. Nine patients ( $\mathrm{n}=4$ females) aged 33-78 years $(M=60.22, S D=13.17)$ participated in the study. They already received different therapeutic treatments as a part of their stroke rehabilitation at T0 was for one up to 32 weeks $(M=11.33, S D=9.15)$. The Barthel Index (BI), a test for evaluation of independence in everyday life in a range of $0-100$ points (scored with 0,5 or 10 points). The participant had an average of 58 points $(S D=13.46)$ and were rated as "need of help". The Fugl-Meyer Score (FMA), a test for evaluation of motor skills in the upper extremities in a range of 0-66 points (scored with 0,1 or 2 points). The participant had an averaged at 54 points $(S D=16.72)$ and the performances were rated as "moderate" impairments.

\section{Questionnaire and data analysis}

The questionnaire applied in the study used questions with a structured, standardized, closed structure. Questions were answered by dichotomous answer options or on five point Likert scales ( $1=$ strongly disagree, $2=$ disagree, $3=$ undecided, $4=$ agree and 5=strongly agree). The items were divided into three areas: UX (e.g. concentration, etc.), TAM (e.g. perceived use, etc.) and potential use at home.

Non-parametric methods were used to analyse the differences between measurements. This procedure was chosen because of small sample size, lack of normal distribution and ordinal response scale. Two-sample paired Wilcoxon signed-rank tests were performed. Because of the small sample size $(n<10)$, one-sided exact tests were used. All statistical tests were performed on a significance level of $\alpha=0.05$. Cohen's $d$ was used as effect size.

\section{Results}

\section{User experience with BMI}

In order to assess the experience of stroke patients trained with the BMI, the subjects were asked to answer various 
questions after using the training device. Table 1 lists medians, $p$-values and effect sizes of various UX factors during training with the BMI and the visual feedback in comparison of T0-T1. According to the medians, it can be seen that above-average positive values were given at both measurement points in the variables of concentration, safety, well-being and motivation during the training as well as all variables for visual feedbacks. At the same time, both measurement points show that fatigue, anxiety, under- and overstrain, boredom and pain were given as rather little or not at all. The analysis shows no statistically significant differences between T0 and T1 in terms of UX ( $p>0.05)$. However, small to large effect sizes can be seen in most variables, which imply a practical relevance of those differences.

\section{Acceptance of the BMI}

The ratings of the four TAM dimensions were assessed on a five-level Likert scale. Table 2 shows the descriptive data, $\mathrm{p}$-values and effect sizes of the measurement points T0 and T1. The medians show that positive values were given in all four dimensions at both measuring points. The variable "intended use" remains constant in the ratings. The ratings for the scales "perceived use", "user-friendliness" and "social norm" decreased marginally at the second measurement point compared to the first. The analysis shows no statistically significant differences between $\mathrm{T} 0$ and $\mathrm{T} 1$ in all TAM dimensions ( $p>0.05$ ). But, small to medium effect sizes were found in all dimensions, which implies a practical relevance of these differences.

Table 1: Statistics for comparison of T0-T1: UX of stroke patients $(n=9)$.

T0 (MED \pm IQB $) \quad$ T1 $($ MED \pm IQB $) \quad$ p-Value

"During the training, I felt ..."

\begin{tabular}{lrrrr} 
Safety & $4.00 \pm 3.0 / 5.0$ & $4.00 \pm 3.0 / 5.0$ & 0.450 & 0.521 \\
Motivation & $5.00 \pm 3.5 / 5.0$ & $4.00 \pm 3.5 / 5.0$ & 0.500 & 0.707 \\
Concentration & $4.00 \pm 4.0 / 5.0$ & $5.00 \pm 4.0 / 5.0$ & 0.156 & 1.069 \\
Well-being & $4.00 \pm 3.0 / 4.5$ & $4.00 \pm 4.0 / 5.0$ & 0.188 & 0.861 \\
Fatigue & $1.00 \pm 1.0 / 2.5$ & $1.00 \pm 1.0 / 2.5$ & 0.500 & 0.301 \\
Overstrained & $1.00 \pm 1.0 / 3.0$ & $1.00 \pm 1.0 / 2.5$ & 0.250 & 0.511 \\
Pain & $1.00 \pm 1.0 / 2.5$ & $1.00 \pm 1.0 / 2.0$ & 0.375 & 0.485 \\
Boredom & $1.00 \pm 1.0 / 2.0$ & $1.00 \pm 1.0 / 2.0$ & 0.500 & 0.707 \\
Understrained & $1.00 \pm 1.0 / 2.0$ & $1.00 \pm 1.0 / 3.0$ & 0.125 & 1.173 \\
Fear & $1.00 \pm 1.0 / 1.0$ & $1.00 \pm 1.0 / 1.0$ & 0.500 & 0.707 \\
“The visual feedback was $\ldots$ & & & \\
Motivating & $4.00 \pm 0.5 / 0.5$ & $3.00 \pm 1.0 / 0.0$ & 0.102 & 1.298 \\
Understandable & $5.00 \pm 0.0 / 1.5$ & $4.00 \pm 1.0 / 0.0$ & 1.0 & 0 \\
Supporting & $5.00 \pm 0.0 / 1.5$ & $5.00 \pm 0.0 / 1.5$ & 1.0 & 0 \\
Satisfying & $4.00 \pm 1.0 / 0.5$ & $4.00 \pm 0.5 / 0.5$ & 0.458 & 0.511 \\
\hline
\end{tabular}

Table 2: Statistics for comparison of T0-T1: TAM of stroke patients $(n=9)$.

\begin{tabular}{lrrrr}
\hline & T0 $($ MED \pm IQB) & T1 $($ MED \pm IQB) & p-Value & $\boldsymbol{d}$ \\
\hline Intended use & $4.00 \pm 1.0 / 1.0$ & $4.00 \pm 1.0 / 1.25$ & 0.313 & 0.463 \\
Perceived use & $4.67 \pm 0.33 / 1.0$ & $4.00 \pm 1.0 / 0.5$ & 0.188 & 0.779 \\
Ease of use & $4.33 \pm 0.27 / 0.83$ & $4.00 \pm 0.5 / 0.5$ & 0.391 & 0.367 \\
Social norm & $4.00 \pm 1.0 / 1.0$ & $3.50 \pm 1.0 / 0.5$ & 0.250 & 0.779 \\
\hline
\end{tabular}

TO

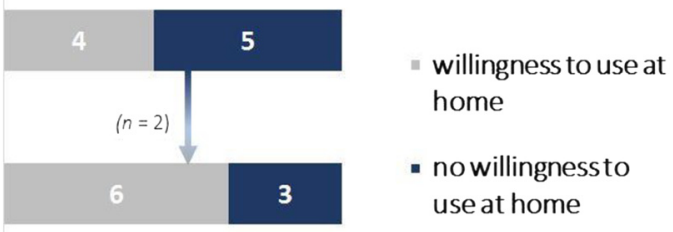

$-1$

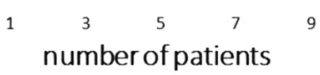

Figure 1: Descriptive data of willingness to use the Bi-Manu-Interact $(B M I)$ at home and migration of patients T0 vs. T1 $(n=9)$.

\section{Potential for home use of the BMI}

The patients were asked whether they could imagine using the BMI at home. The answer was given dichotomously with "yes" or "no". Figure 1 shows that the willingness to use the BMI at home increased at T1 compared to T0. At TO four patients could imagine use the BMI at home. At T1 the willingness increased to six affirmations. The data show a migration of two patients who did not show willingness at T0. After 10 training sessions (T1) they could imagine to use the BMI at home.

\section{Conclusion}

This paper presents results of a pilot field study aimed to detect long-term changes in TAM, UX and the potential of home use over 10 therapy sessions (T0-T1) with the experimental demonstrator BMI in stroke rehabilitation. The results are answering our research question and hypotheses.

H1: The descriptive data shows that the ratings of stroke patients in all variables of UX during the training show no change to T1. Except for motivation, there is a slight decrease from T0 to T1. Also the experience that visual feedback is perceived as motivating decreases from $\mathrm{T} 1$ to T0. But the difference cannot be confirmed statistically. Thus HO will be rejected. 
H2: The descriptive data shows that the variable of intended use remains constant. Perceived use, ease of use and social norm decrease marginal for the second measurement. The differences in the measuring times cannot be statistically verified, so $\mathrm{HO}$ will also be rejected.

H3: The willingness to use the BMI at home increased at T1 compared to T0. There is a migration of two patients from T0 to T1, so H3 can be accepted. The differences in measurement times cannot be statistically verified due to the small number of subjects. Further studies with larger sample sizes must follow to confirm this trend.

The results of the study suggest that familiarity gained through repetitive training sessions with the BMI. Presumably the increased experience with the BMI shows a higher rating on concentration and the potential to use the BMI at home. The findings are coherent with previous studies, based on the UX lifecycle model [17] it can be assumed that long-term use with an interactive device will result in a change of usage effects e.g. learning and habituation through repeated usage. The findings in TAM are also coherent with previous studies [15, 16]. An increased experience in the use of a device leads to a decrease in the dimensions perceived use and the intended use. This is due to the advantages and disadvantages of a technology are perceived in a more differentiated way. According to previous findings [18] the data shows that the training with the BMI already shows a change in the clinic to be used in the home environment of the patient. In this way, patients can become familiar with the therapy system under the supervision of the therapists.

Acknowledgment: We would like to thank the Brandenburg Klinik, Bernau for their clinical support. Also, we would like to sincerely thank all patients and therapists for participating in the study.

Research funding: Natalie Jankowski and Lea Wiehe were supported by the grant from Federal Ministry of Education and Research of Germany. (BeMobil: FKZ 16SV7071).

Author contributions: All authors have accepted responsibility for the entire content of this manuscript and approved its submission.

Conflict of interest: Authors state no conflict of interest.

Informed consent: Informed consent has been obtained from all individuals included in this study.

Ethical approval: The research related to human use complies with all the relevant national regulations, institutional policies and was performed in accordance with the tenets of the Helsinki Declaration.

\section{References}

1. WHO. Häufigste Todesursachen weltweit im Jahr 2016. Statista 2018 (in Millionen) [Graph].

2. Langhorne P, Coupar F, Pollock A. Motor recovery after stroke: a systematic review. Lancet Neurol 2009;8:741-54.

3. Langhorne P, Bernhardt J, Kwakkel G. Stroke rehabilitation. Lancet 2011;377:1693-702.

4. Riener R, Disselhorst-Klug C, Schmidt H, Nef T. Therapie-und Assistenzsysteme für die Bewegungsrehabilitation. In: Biomedizinische Technik: Band 10 Rehabilitationstechnik. Berlin: DeGruyter; 2015:445-96.

5. Vanoglio F, Bernocchi P, Mulè C, Garofali F, Mora C, Taveggia G, et al. Feasibility and efficacy of a robotic device for hand rehabilitation in hemiplegic stroke patients: a randomized pilot controlled study. Clin Rehabil 2017;31:351-60.

6. Zhang C, Li-Tsang CW, Au RK. Robotic approaches for the rehabilitation of upper limb recovery after stroke: a systematic review and meta-analysis. Int J Rehabil Res 2017;40:19-28.

7. Lo K, Stephenson M, Lockwood C. Effectiveness of robotic assisted rehabilitation for mobility and functional ability in adult stroke patients: a systematic review. JBI Database Syst Rev Implement Rep 2017;15:3049-91.

8. Molier BI, Van Asseldonk EH, Hermens HJ, Jannink MJ. Nature, timing, frequency and type of augmented feedback; does it influence motor relearning of the hemiparetic arm after stroke? A systematic review. Disabil Rehabil 2010;32:1799-809.

9. Colomer C, Llorens R, Noé E, Alcañiz M. Effect of a mixed realitybased intervention on arm, hand, and finger function on chronic stroke. J Neuro Eng Rehabil 2016;13:45.

10. Ivanova E, Krause A, Schälicke M, Schellhardt F, Jankowski N, Achner J, et al. Let's do this together: bi-manu-interact, a novel device for studying human haptic interactive behavior. In: International conference on rehabilitation robotics (ICORR). IEEE; 2015:708-13. https://doi.org/10.1109/ICORR.2017.8009331.

11. Jordan PW. Designing pleasurable products: an introduction to the new human factors. London: CRC Press; 2003.

12. Minge M. Dynamische Aspekte des Nutzungserlebens der Interaktion mit technischen Systemen [Ph.D. dissertation]. TU Berlin Zentralbibliothek: Technische-Universität Berlin; 2011.

13. Marcellini F, Mollenkopf H, Spazzafumo L, Ruoppila I. Acceptance and use of technological solutions by the elderly in the outdoor environment: findings from a European survey. Zeitschrift Gerontologie geriatrie 2000;33:169-77.

14. Davis FD, Bagozzi RP, Warshaw PR. User acceptance of computer technology: a comparison of two theoretical models. Manag Sci 1989;35:982-1003.

15. King WR, He J. A meta-analysis of the technology acceptance model. Inf Manag 2006;43:740-55.

16. Claßen K. Zur Psychologie von Technikakzeptanz im höheren Lebensalter: Die Rolle von Technikgenerationen [Ph.D. dissertation]: Karl-Rupprechts- Universität Heidelberg; 2013. https://doi.org/10.11588/heidok.00014295.

17. John M, Einhaus J, Klose S, Kock G, Graßhoff T. Telerehabilitation 2015 - Medizinische Assistenzsysteme in der Prävention, Rehabilitation und Nachsorge. Berlin: Fraunhofer-Public, 2015.

18. Pohlmeyer AE, Hecht M, Blessing L. Use experience lifecycle model contin UE. Berlin, Germany: Proc BWMMS; 2009:314-7. 